\title{
Analysis on Steering Mechanics Model of Tracked Tank Cleaning Robot
}

\author{
Meng Fanzhao ${ }^{1,2, a}$, Chen Fude ${ }^{1,2, b}$ and Tan Dening 1, 2, c \\ ${ }^{1}$ School of Mechanical Engineering, Shandong Huayu University of Technology, Dezhou 253034, China \\ ${ }^{2}$ Dezhou Municipal Key Laboratory of Industrial Robot Control, Dezhou 253034, China \\ a mfzhthy@163.com, b chenfude2008@126.com, ㄷdn2013@163.com
}

Keywords: tracked robot, mathematical model, steering mechanics

\begin{abstract}
The physical model of a tracked tank cleaning robot that works on the sludge ground is set up, which provides a theoretical basis for optimizing the chassis design of the tank cleaning robot. In the modelling process, the sludge resistance of the track and the ground friction are taken into account, and the slip speed is introduced, and the steering torque during the course of the track is obtained. Finally, according to the design of the tracked robot and the related parameters of its working environment, the optimal design is used to obtain the optimum track width, grounding contact length, and the distance between two tracks. Theoretical analysis and simulation proves that the steering mechanics model is correct and effective.
\end{abstract}

\section{Introduction}

The tank cleaning robot is mainly composed of a moving mechanism and a driving system, as shown in Figure 1. The tracked walking mechanism is used, because it has the advantages of simple structure, strong ground adaptability, and easy control [1]. In the past, the design of tracked robot walking mechanism mainly focused on its structural design, and whether the strength and stiffness of its structure could meet the design requirements, less attention was paid to the research on the mechanical properties of tracked ground [2]. The track's mechanical properties often affect the robot's energy consumption, mobility, and even smooth walking [3]. In general, the ground contact length, width, and distance between the tracks are the main parameters that constitute the mechanics of the track surface. It can also be known that in the case of a fixed track ground length, there is an optimum track width so that the ratio of the minimum track resistance ground contact length to the flexibility of the turn. Therefore, the track and sludge mechanics has important significance for the design of tracked walking mechanism. Tracks on the sludge ground to turn to overcome friction in addition to overcome the track to push sludge resistance. At present, the analysis of the mechanical model of track walking steering is not comprehensive, only the solution of the friction between the track and the ground under the hard road conditions is analyzed, or only the solution of the bulldozing resistance under soft road conditions is considered, there is no general mathematical model [4]. The purpose of this paper is to create a mathematical model of the track robot's steering on the sludge ground, and this model is applied to the tank cleaning robot design.

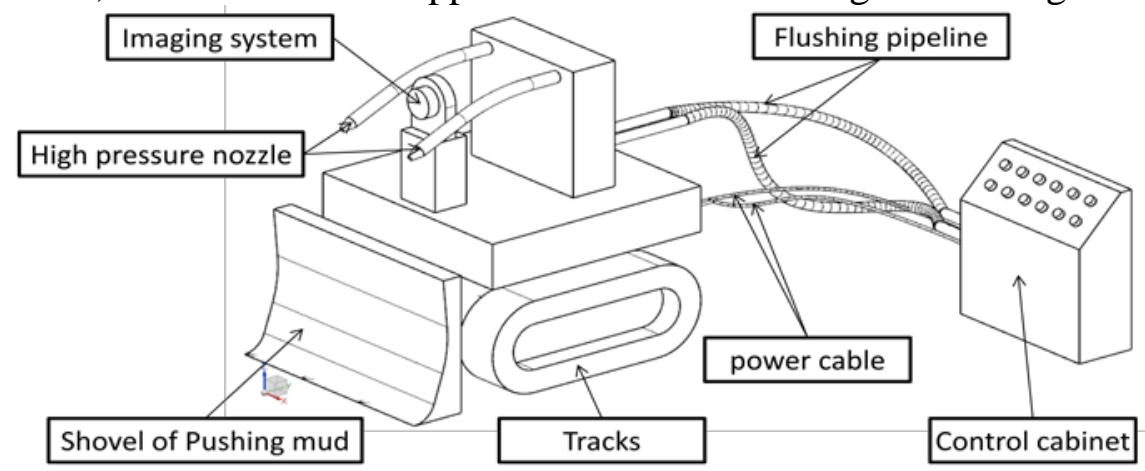

Figure1 Tracked tank cleaning robot model 


\section{Turning analysis}

\subsection{Physical model of robot}

When the tracked tank cleaning robot walks on the sludge of the tank, it only needs to overcome the friction between the track and the sludge. In the steering, the track slides relatively to the sludge ground, mainly to overcome the friction between the track and the sludge pushing resistance, and the physical models when it is steering situation are shown in Figure 2. The turning radius $R_{o}$ and angular velocity $\omega_{o}$ are solved by Equation (1).

$$
\left.\begin{array}{l}
R_{O}=\frac{\mid \frac{v_{a 2}+v_{a 1} \mid}{\left|v_{a 2}-v_{a 1}\right|} \cdot \frac{B}{2}}{v_{0}} \\
\omega_{O}=\frac{v_{a 2}-v_{a 1}}{B}
\end{array}\right\}
$$

Where, $B$ is the center distance of two tracks, and $v_{a 1}$ and $v_{a 2}$ are speeds of two tracks respectively. However, in the actual process, since there are slips between the tracks and lateral offsets occur at the same time [5][6], the turning process is not an ideal situation as shown in Figure2. In order to make the model closer to the reality, the derived mathematical model is more general, and the physical model of the tracked turning is modified. In order to improve the model compliance, the slip speeds are introduced. In order to simplify the calculation, the slip speeds on both sides of the tracks are given as $\delta$.

$$
\left.\begin{array}{c}
R=\frac{\left|v_{a 2}+v_{a 1}\right|}{\left|v_{a 2}-v_{a 1}-2 \delta\right|} \cdot \frac{B}{2} \\
\omega_{O}=\frac{v_{a 2}-v_{a 1}-2 \delta}{B}
\end{array}\right\}
$$

The horizontal displacement generated by the instantaneous center of velocity of the tracked ground is defined as $A_{i}$, and the longitudinal displacement is $D_{i}, \quad(i=1,2, i=1$ for inner side, $i=2$ for outer side). Let $A_{1}=A_{2}=A, D_{1}=D_{2}=D$, therefore, the actual track turning model is shown in Figure 3.

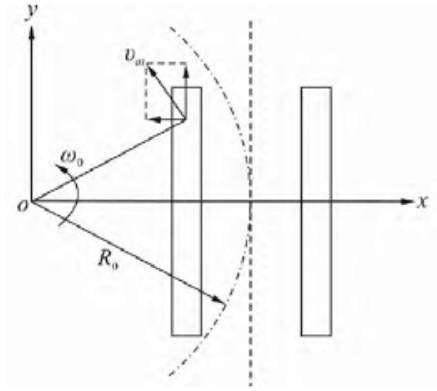

Figure2 Ideal track turning model

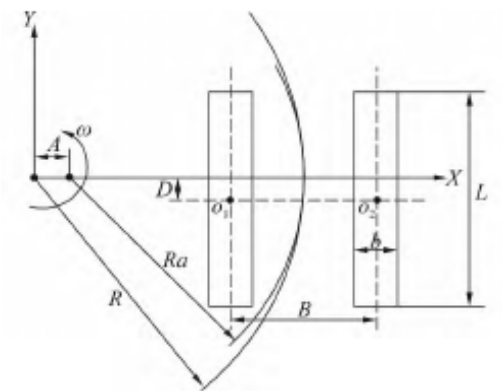

Figure3 Track real turning model

\subsection{Mathematical model}

\subsubsection{Calculation of frictional resistance between track and sludge}

With a caterpillar as the object of analysis, the instantaneous center velocity of the track is used as the origin, and the coordinate system are shown in Figure 4. Take a microelement $d x d y$ of the track ground, and there is trace friction $d_{F_{i}}$ (where $i=1$, 2, on behalf of the inner and outertrack), the direction is opposite to the absolute speed of the point, there are:

$$
d_{F_{i}}=\mu \cdot p(x, y) d x d y
$$

Where $p(x, y)$ is the ground pressure function. The components of $d_{F}$ in the $X$-axis and $Y$-axis directions are: 


$$
\left.\begin{array}{l}
d_{F_{x i}}=-d_{F_{i}} \cdot \sin \sin \varphi=-d_{F_{i}} \cdot \frac{y}{\sqrt{x^{2}+y^{2}}} \\
d_{F_{y i}}=d_{F_{i}} \cdot \cos \cos \varphi=d_{F_{i}} \cdot \frac{x}{\sqrt{x^{2}+y^{2}}}
\end{array}\right\}
$$

From Equation (4), it can see that the change in the steering resistance $M$ (around the track velocity instant center $O$ ) of the track are:

$$
M_{O i}=\iint_{y \Phi F_{x i}}-x d F_{y i}=\int_{y_{i 1}}^{y_{i 2}} \int_{x_{i 1}}^{x_{i 2}}\left[\frac{x^{2}+y^{2}}{\sqrt{x^{2}+y^{2}}}\right] \mu p(x, y) d x d y
$$

\subsubsection{Track side sludge resistance calculation}

If the quality of the sludge on the side is neglected, the force on the side of the track is shown in Figure5. Where $Q$ is the reaction force of the lower sludge on the wedge sludge, $C_{\theta}=Z C / \sin \theta$ is the cohesion per unit area, $W$ is the sludge weight per unit area, $R_{B}(\theta)$ is the sludge resistance per unit length, $\phi_{\omega}$ is the friction angle of the wall, $\theta$ is the angle of the failure surface, and $\varphi$ is the inner friction angle of the oil sludge. According to the relationship between the amount of load sinking [7], Equation (6) can be derived as:

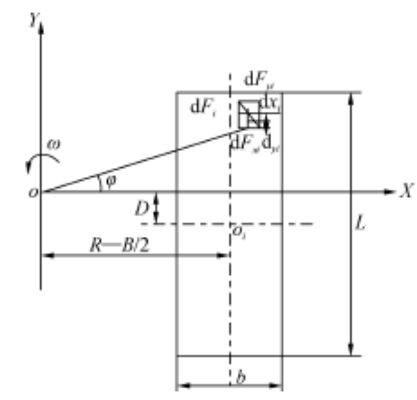

Figure4 Force diagram of track by the ground

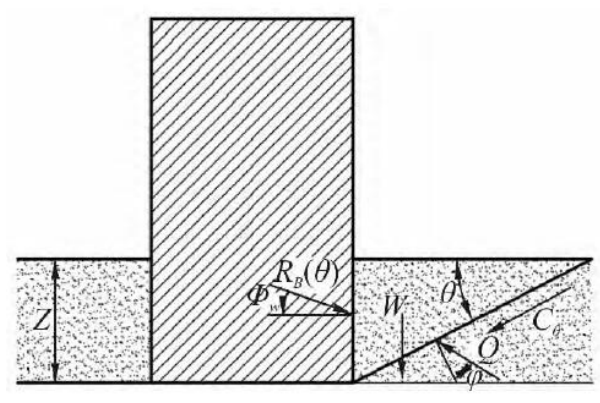

Figure5 Force diagram of track by side

$$
z=\left[\frac{F_{z i}}{2 L b\left(\frac{k_{C}}{b}+k_{\varphi}\right)}\right]^{\frac{1}{n}}
$$

Where $\mathrm{z}$ is the amount of subsidence, $k_{c}, k_{\varphi}$ are the cohesive and frictional deformation modulus of sludge, and $\mathrm{n}$ is the deformation index. The pushing sludge resistance $R_{B}$ of any unit length on both sides of the track can be obtained from the force balance.

$$
\left.\begin{array}{l}
\sum Y=R_{B}(\theta) \cos \cos \phi_{\omega}+z c \cot \cot \theta-Q \sin \sin (\theta+\varphi)=0 \\
\sum Z=R_{B}(\theta) \sin \sin \phi_{\omega}+z c-\gamma_{S} z^{2} \cot \cot \theta / 2-Q \cos \cos (\theta+\varphi)=0 \\
R_{B}(\theta)=\frac{\gamma_{S^{2}}{ }^{2} \cot \cot \theta / 2+z C[1+\cot \cot \theta \cot \cot (\theta+\varphi)]}{\cos \cos \phi_{\omega} \cos \cos (\theta+\varphi)-\sin -\sin \phi_{\omega}}
\end{array}\right\}
$$

Where $\gamma_{s}$ is the sludge bulk density. Since $M_{T i}$ is only a function of $\theta$, the minimum value of $M_{T i}$ corresponds to a certain value of $\theta$, at this value of $\theta$, the sludge ground is destroyed, so the steering resistance torque generated by the lateral pushing sludge resistance is

$$
\begin{gathered}
M_{T i}=\int_{-(\mathrm{L} / 2+D)}^{0} R_{B}(\theta) \cos \cos \phi_{\omega}(-y) d y+\int_{0}^{\mathrm{L} / 2-D} R_{B}(\theta) \cos \cos \phi_{\omega} y d y-F_{y i}^{\prime} D \\
F_{y i}^{\prime}=-\int_{0}^{\mathrm{L} / 2-D} R_{B}(\theta) \cos \cos \phi_{\omega} d y+\int_{-(\mathrm{L} / 2+D)}^{0} R_{B}(\theta) \cos \cos \phi_{\omega} d y=R_{B}(\theta) \cos \cos \phi_{\omega} 2 D
\end{gathered}
$$


Substituting Equation (9) into Equation (8), it is obtained:

$$
M_{T i}=\cos \cos \phi_{\omega}\left(\frac{L^{2}}{4}-D_{i}^{2}\right) \times \frac{\gamma_{S} z^{2} \cot \cot \theta / 2+z C[1+\cot \cot \theta \cot \cot (\theta+\varphi)]}{\cos \cos \phi_{\omega} \cos \cos (\theta+\varphi)-\sin -\sin \phi_{\omega}}
$$

Ignoring the factors of the internal frictional resistance of the track, the total steering resistance torque $\mathrm{M}$ caused by the sludge ground is:

$$
M=\sum M_{O i}+\sum M_{T i}
$$

\subsubsection{Mathematical model of tracked robot on the sludge ground}

According to the Equation (3)-(11), a general mathematical model of tracked robot on the sludge ground are shown in Equation (12). When the tracked tank cleaning robot works normally, it must be able to satisfy the steering conditions, that is, for a uniform rotation, the driving torque is greater than the preventing steering torque.

$$
\begin{aligned}
& \min M=\int_{-\mathrm{L} / 2-D}^{\mathrm{L} / 2-D} \int_{R+\mathrm{B} / 2-0.5 b}^{R+\mathrm{B} / 2+0.5 b}\left[\frac{x^{2}+y^{2}}{\sqrt{x^{2}+y^{2}}}\right] \mu p(x, y) d x d y \\
& +\int_{-\mathrm{L} / 2-D}^{\mathrm{L} / 2-D} \int_{R-\mathrm{B} / 2-0.5 b}^{R-\mathrm{B} / 2+0.5 b}\left[\frac{x^{2}+y^{2}}{\sqrt{x^{2}+y^{2}}}\right] \mu p(x, y) d x d y \\
& +\sum_{i=1,2} \cos \phi_{\omega}\left(\frac{L^{2}}{4}-D_{i}^{2}\right) \times \frac{\gamma_{S} z^{2} \cot \cot \theta / 2+z C[1+\cot \theta \cot (\theta+\varphi)]}{\cos \phi_{\omega} \cos (\theta+\varphi)-\sin \phi_{\omega}}
\end{aligned}
$$

The driving torque is:

$$
M\left(F_{y i}\right)=\iint_{x} d F_{y i}=\int_{x_{i 1}}^{x_{i 2}} \int_{y_{i 1}}^{y_{i 2}}\left[\frac{x^{2}}{\sqrt{x^{2}+y^{2}}}\right] \mu p(x, y) d x d y
$$

The preventing steering torque is :

$$
M\left(F_{x i}\right)=\iint y d F_{x i}=\int_{x_{i 1}}^{x_{i 2}} \int_{y_{i 1}}^{y_{i 2}}\left[\frac{\mathrm{y}^{2}}{\sqrt{x^{2}+y^{2}}}\right] \mu p(x, y) d x d y
$$

The necessary condition for the successful steering of the tracked robot is:

$$
\sum_{i=1}^{2}\left|M\left(F_{y i}\right)\right|>\sum_{i=1}^{2}\left|M\left(F_{x i}\right)\right|+\left|M_{T}\right|
$$

\section{Conclusions}

The mathematical models of the tracked robot's steering on the sludge ground are deduced. The minimum torque of steering is calculated, and then the best value of the width of the track, the length of the ground and the distance between tracks are also obtained. At the same time, the minimum driving torque is calculated. It provides a theoretical basis for optimizing the chassis design of the tracked tank cleaning robot.

\section{References}

[1] Kwon H J, Shim H, Kim D, et al. (2007) A development of a transformable caterpillar equipped mobile robot. //IEEE International Conference on Control, Automation and Systerms, 1062-1065.

[2] Zhao Yu, Yan Hongwei. (2010) Design analysis and research on tracked walking mechanism. New Technology \& New Process, 5, 50-53.

[3] Liu Guomin, Huang Haidong, Zhang Cui. (2010) Analysis on turning mode for crawler chasses. Chinese Journal of Construction Machinery, 8(3),309-313. 
[4] Wong J Y. (2009) Development of high-mobility tracked vehicles for over snow operations. Journal of Terramechanics, 46(4), 141-155.

[5] Cheng Junwei, Gao Lianhua, Wang Hongyan, et al. (2007) Calculation of tracked vehicle steering-load based on conditions of tracks' slip and track-width considered. Vehicle \& Power Technology, 1,40-44.

[6] Shi Lichen, Wang Liangxi, Zhang Bingzhi. (2003) Dynamic simulation of tracked vehicle steering performance. Acta Armamentarii, 24(3), 289-293.

[7] Liu Guomin, Huang Haidong. (1998) Study on turning track of caterpillar base plate. Water Conservancy \&Electric Power Machinery, 5, 18-21. 